ARTICLE

\title{
Structural basis for the transition from translation initiation to elongation by an 80S-elF5B complex
}

Jinfan Wang (1) ${ }^{1}$, Jing Wang ${ }^{2}$, Byung-Sik Shin ${ }^{3}$, Joo-Ran Kim³ ${ }^{3}$, Thomas E. Dever (D) ${ }^{3 凶}$, Joseph D. Puglisi (D) ${ }^{1 凶} \&$ Israel S. Fernández ${ }^{2 凶}$

Recognition of a start codon by the initiator aminoacyl-tRNA determines the reading frame of messenger RNA (mRNA) translation by the ribosome. In eukaryotes, the GTPase elF5B collaborates in the correct positioning of the initiator Met-tRNA $A_{i}$ Met on the ribosome in the later stages of translation initiation, gating entrance into elongation. Leveraging the long residence time of elF5B on the ribosome recently identified by single-molecule fluorescence measurements, we determine the cryoEM structure of the naturally long-lived ribosome complex with elF5B and Met-tRNA $A_{i}$ Met immediately before transition into elongation. The structure uncovers an unexpected, eukaryotic specific and dynamic fidelity checkpoint implemented by eIF5B in concert with components of the large ribosomal subunit.

\footnotetext{
${ }^{1}$ Department of Structural Biology, Stanford University School of Medicine, Stanford, CA, USA. ${ }^{2}$ Department of Biochemistry and Molecular Biophysics, Columbia University, New York City, NY, USA. ${ }^{3}$ Eunice Kennedy Shriver National Institute of Child Health and Human Development, NIH, Bethesda, MD, USA. ${ }^{凶}$ email: thomas.dever@nih.gov; puglisi@stanford.edu; isf2106@cumc.columbia.edu
} 
$\mathrm{P}$ rotein synthesis starts with the assembly of a ribosomal complex at the start site on the mRNA ${ }^{1}$. Eukaryotes employ numerous translation initiation factors (eIFs) to achieve the 80 S initiation complex (IC) with the initiator aminoacyl-tRNA (Met-tRNA ${ }_{\mathrm{i}}{ }^{\mathrm{Met}}$ ) and the AUG start codon of the mRNA programmed in the ribosomal peptidyl-tRNA site (P site) $)^{2}$. The $40 \mathrm{~S}$ small ribosomal subunit, accompanied by eIFs and MettRNA ${ }_{i}{ }^{M e t}$, is recruited to the $5^{\prime}$-untranslated region (5'-UTR) of the 7-methylguanosine-capped $\mathrm{mRNA}^{3}$. This is followed by a dynamic inspection of the $5^{\prime}$-UTR in search of the correct AUG codon as a start site, a process termed "scanning"4,5. Upon AUG recognition by the Met-tRNA ${ }_{i}$ Met anticodon, a series of eIF reorganizations and departures coupled to ribosomal conformational rearrangements occurs, resulting in a post-scanning $48 \mathrm{~S}$ preinitiation complex (PIC) ${ }^{6-8}$. This 48 S PIC is then joined by the 60 S large ribosomal subunit to form the 80S IC, catalyzed by the universally conserved GTPase eIF5B (Fig. 1a) ${ }^{9,10}$. Using singlemolecule fluorescence methods, we recently revealed that progression of the Saccharomyces cerevisiae 80S IC to the elongationcompetent state (80S EC) with an exposed codon in the A site for aminoacyl-tRNA delivery is gated by the slow eIF5B dissociation from the complex (Supplementary Fig. 1a, b) ${ }^{11}$. This dissociation
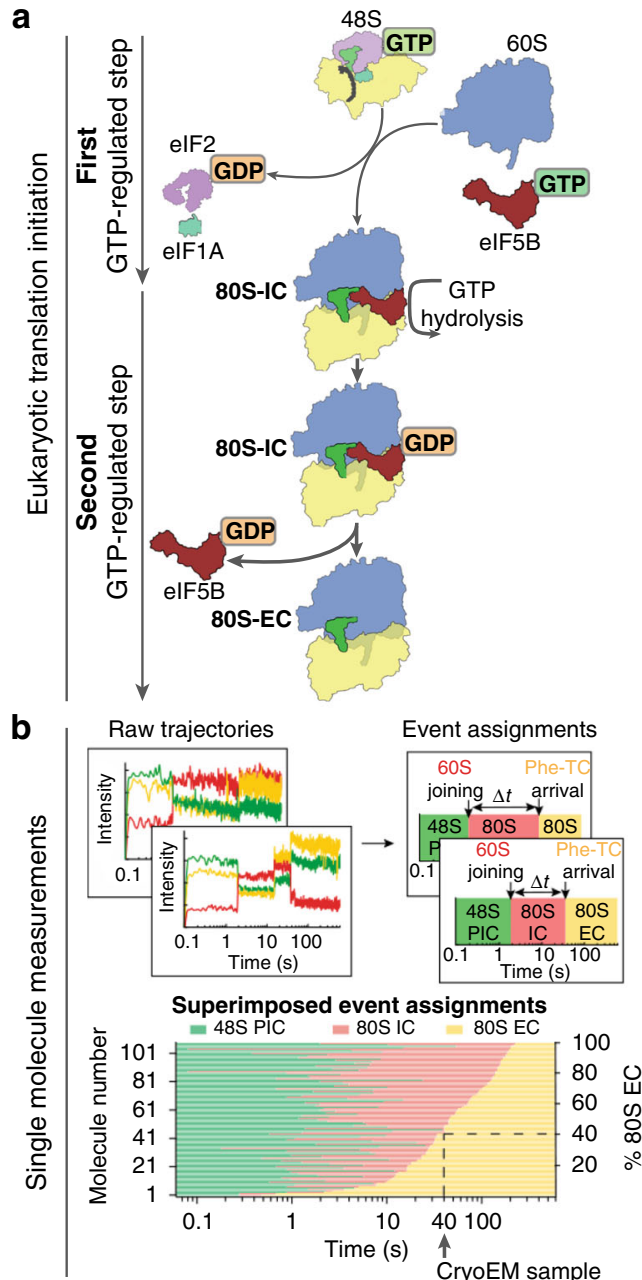

of eIF5B requires GTP hydrolysis, with the timing of its dissociation critical to stringent start-codon selection.

These dynamics results left unanswered whether the slow dissociation of eIF5B was limited by its GTPase activation/GTP hydrolysis, Pi release, and/or eIF5B:GDP dissociation. Previous cryo-EM reconstructions have described the architectures of the 80 S IC bound with eIF5B:GDPCP at medium resolution, representing snapshots of the assembly prior to eIF5B GTP hydrolysis $^{12,13}$. In addition, Met-tRNA $A_{i}$ Met has sequence features that allow it to bind directly to the $\mathrm{P}$ site of the ribosome during initiation, unlike elongator tRNAs ${ }^{14-16}$. Here, guided by the kinetics determined by our previous single-molecule fluorescence measurements, we performed cryo-EM analysis of the onpathway initiation complexes to provide high-resolution information on the molecular mechanism by which eIF5B assists on the positioning of the initiator tRNA into the ribosomal P site and gates the transition from initiation to elongation.

\section{Results and discussion}

Architecture of the eIF5B-bound 80S IC. Leveraging the slow dissociation of eIF5B from the 80S IC in the native initiation

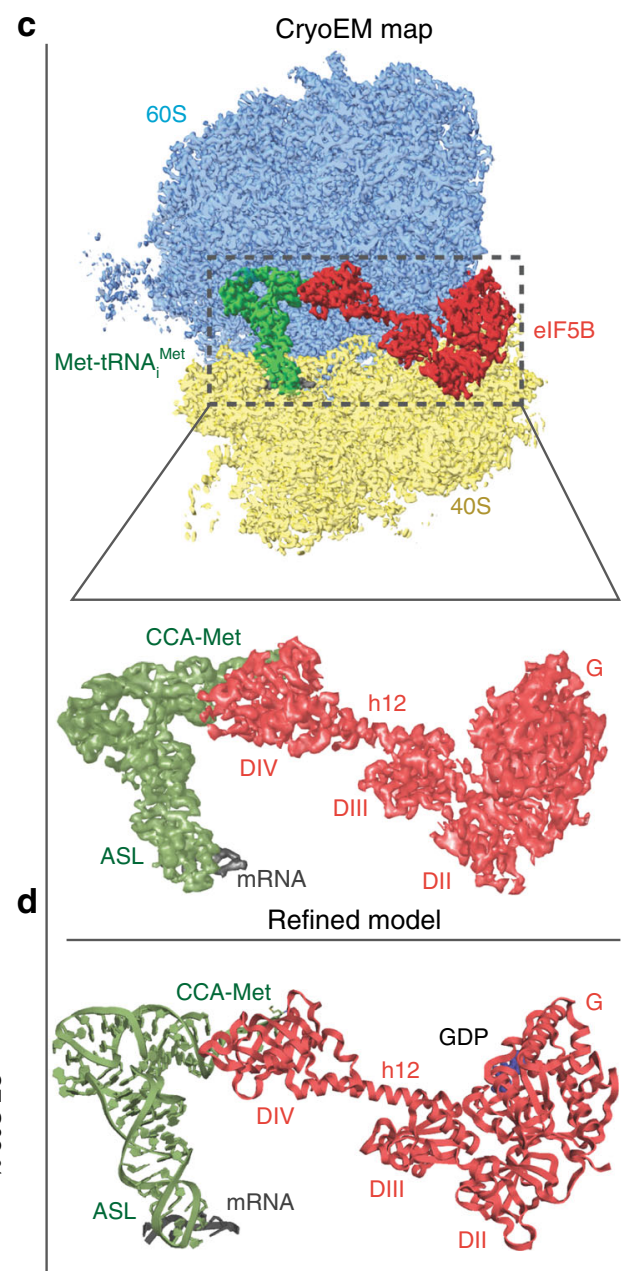

Fig. 1 Cryo-EM sample preparation guided by single-molecule fluorescence data and overall architecture. a Overview of translation initiation in eukaryotes. Two steps, regulated by GTPases elF2 and elF5B control the delivery of Met-tRNA $A_{i}$ Met. $\mathbf{b}$ Cryo-EM samples were prepared and froze at the timepoint $40 \mathrm{~s}$ after mixing $48 \mathrm{~S}$ PICs, elF5B:GTP, and 60S. At this timepoint, $\sim 40 \%$ of the total $80 \mathrm{~S}$ particles are expected to be $80 \mathrm{~S}$ EC, while the other $\sim 60 \%$ are $80 \mathrm{~S} \mathrm{IC}$ with elF5B-bound, as demonstrated by our previous single-molecule fluorescence data (Supplementary Fig. 1) ${ }^{11}$. c Overview of the final cryo-EM map obtained after maximum likelihood classifications in Relion ${ }^{44}$. Large subunit (60S) is colored in blue, small subunit (40S) yellow, MettRNA ${ }_{i}$ Met green, mRNA gray, and elF5B red. Bottom, details of the local map obtained for Met-tRNA $A_{i}$ Met, mRNA, and elF5B. Domain IV (DIV) of elF5B is in close contact with the acceptor stem of Met-tRNA ${ }_{i}^{\text {Met }}$. $\mathbf{d}$ Stereochemically refined models for Met-tRNA $A_{i}$ Met, $m R N A$, and elF5B with components indicated. 
pathway (average lifetime of the eIF5B-bound $80 \mathrm{~S}$ state is $30-60 \mathrm{~s}$ at $\left.20^{\circ} \mathrm{C}\right)^{11}$, we prepared and froze samples at a pre-steady-state reaction timepoint ( $40 \mathrm{~s}$, Fig. 1b, Supplementary Fig. 1, and "Methods") corresponding to when $\sim 60 \%$ of the assembled $80 \mathrm{~S}$ should be bound with eIF5B (80S IC) after mixing 48S PICs with eIF5B:GTP and 60S subunits. Image processing and 3D classification in Relion $3^{17,18}$ identified a homogeneous class of $~ 70 \%$ of the total pool of $80 \mathrm{~S}$ particles, allowing the reconstruction of a $3 \mathrm{D}$ map with a global resolution of $2.9 \AA$ (Supplementary Figs. 2-4). The reconstruction shows clear density for a tRNA in the P-site region and density for all domains of eIF5B (Fig. 1c). Further analysis of the map revealed excellent densities for domain III (DIII), the connecting $\alpha$-helix 12 (Fig. $1 \mathrm{c}, \mathrm{d}, \mathrm{h} 12^{19}$ ) and especially for domain IV (DIV) of eIF5B, as well as for the Met-tRNA ${ }_{i}$ Met and the start codon of the mRNA (Fig. 1c, d, and Supplementary Fig. 4). Less well-resolved were domains II and G of eIF5B and the elbow region of Met-tRNA ${ }_{i}$ Met (Supplementary Fig. 3).

eIF5B DIV stabilizes the acceptor stem of Met-tRNA ${ }_{i}{ }^{M e t}$ near the PTC. In our reconstruction, DIV of eIF5B is tightly packed against the peptidyl transferase center (PTC) of the large subunit where it contacts the ${ }_{73} \mathrm{ACCA}_{76}$-Met end of Met-tRNA $\mathrm{A}_{\mathrm{i}}$ Met (Fig. 2). The close interaction of eIF5B DIV with the acceptor stem of Met-tRNA ${ }_{i}$ Met enforces a change in the trajectory of the ${ }_{73} \mathrm{ACCA}_{76}$-Met end when compared with the position adopted by the acceptor stem on an elongation peptidyl-tRNA in a canonical configuration (Supplementary Movie 1) ${ }^{20}$. This distortion is not limited to the ${ }_{73} \mathrm{ACCA}_{76}-\mathrm{Met}$ of $\mathrm{tRNA}_{\mathrm{i}}$ Met, as the whole acceptor stem is distorted compared with its position in an elongation tRNA (Fig. 2b, c). The cluster of G-C base pairs in the acceptor stem specific to Met-tRNA ${ }_{i}{ }^{\text {Met }}$ seems to play a pivotal role in this context, allowing a specific distortion of this stem as the MettRNA $_{i}$ Met simultaneously interacts with eIF5B and the start codon (Fig. 2a, b, and Supplementary Fig. 5). Basic residues of the domain IV of eIF5B make specific interactions with this G-C base-pairs cluster ${ }^{21}$; Arg955 of eIF5B contacts the base of G70 of Met-tRNA ${ }_{\mathrm{i}}{ }^{\text {Met }}$ from the major groove of the acceptor stem (Fig. 2e, f). Although mutating Arg955 to Ala in eIF5B did not substantially alter the growth rate of yeast in rich medium, the mutant strain could not grow under amino acid starvation conditions (Fig. 2g), suggesting impaired GCN4 expression. The GCN4 mRNA contains four upstream open reading frames. Translation of the first uORF (uORF1) enables regulated reinitiation at the subsequent uORFs to control GCN4 expression. The Arg955 to Ala mutation in eIF5B significantly impaired derepression of GCN4-lacZ reporter expression under starvation conditions (Fig. 2h), consistent with the growth defect conferred by this mutation under these conditions. Moreover, using a reporter in which the leader was altered and UORF1 was extended to overlap and thus block GCN4 expression (Fig. 2i), the mutation was found to increase the frequency of ribosomes scanning past the start codon (termed "leaky scanning") of uORF1 and translating GCN4. Thus, the interaction between eIF5B Arg955 and Met-tRNA ${ }_{i}{ }^{\text {Met }}$ G70 may also play an important role in start-site selection, and the enhanced leaky scanning of the stimulatory uORF1 might partially account for the defect in GCN4 expression. Consistently, deletion of eIF5B DIV also enhanced leaky scanning and blocked GCN4 expression in yeast ${ }^{19}$ (Fig. $2 \mathrm{~h}$, i).

A eukaryotic specific Met-tRNA ${ }_{i}{ }^{\text {Met }}$ recognition mechanism. The high quality of the map around the PTC region revealed specific contacts of residues of eIF5B DIV with the four-terminal bases of the Met-tRNA $\mathrm{A}_{\mathrm{i}}$ Met as well as with the ribose and phosphate backbone atoms (Fig. 3). Specifically, the base of Met$\mathrm{tRNA}_{\mathrm{i}}{ }^{\text {Met }}$ nucleotide A76 is tightly monitored by eIF5B residues a c

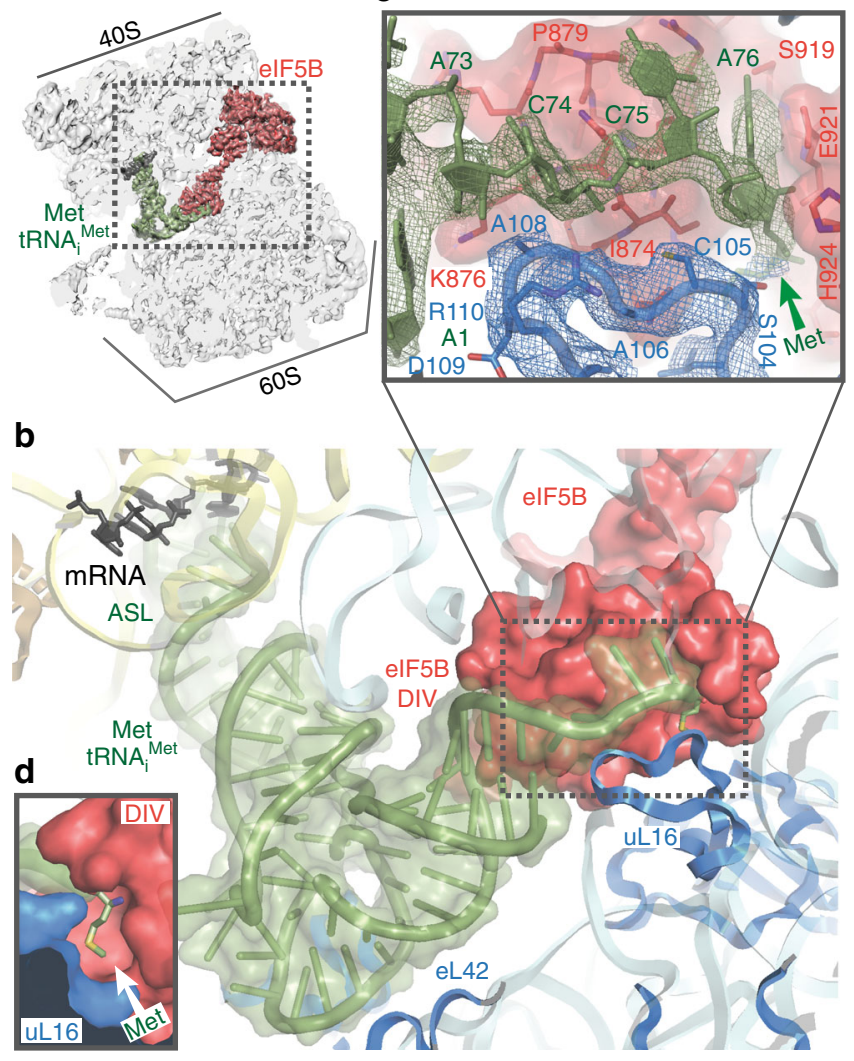

Fig. 3 Ribosomal protein uL16 collaborates with elF5B DIV in MettRNA $\mathbf{A}_{\mathbf{i}}$ Met A76-Met recognition. a Overall view of the 80S/Met-tRNA $\mathrm{A}_{i} \mathrm{Met}$ / elF5B complex in an orientation centered on elF5B DIV. $\mathbf{b}$ A detailed view of the Met-tRNA $A_{i}$ Met conformation adopted while simultaneously interacts with the mRNA and elF5B DIV on the 80S. c A detailed view of elF5B DIV focused on the acceptor stem region of Met-tRNA ${ }_{i}{ }^{\text {Met. }}$. DIV of elF5B is shown in red as semi-transparent $V$ an der Waals surface, Met-tRNA $A_{i}$ Met nucleotides depicted in green with experimental cryo-EM density shown and UL16 residues are in blue with experimental cryo-EM density shown. d DIV of elF5B and residues 102-110 of uL16 form a hydrophobic cavity where the methionine residue of Met-tRNA ${ }_{i}$ Met is hosted.

Glu921 and His924, which anchors the adenine moiety to eIF5B DIV (Fig. 3b, c). In this orientation, the methionyl group esterified to the $3^{\prime} \mathrm{OH}$ of the A76 ribose is directed towards a hydrophobic "pocket" formed by the surface of eIF5B around residue Ile874 (Fig. 3c, d, and Supplementary Fig. 4c).

Intriguingly, this "hydrophobic pocket" is capped by a loop of ribosomal protein uL16, a component of the 60S (Fig. 3d), which is conserved in yeast and humans (Supplementary Fig. 6). This uL16 loop, formed by residues 100 to 120 , is disordered in reported elongation $80 \mathrm{~S}$ complexes, but is well-resolved in our reconstruction, allowing its modeling and refinement. Notably, this loop is stringently checked at the last step of 60S biogenesis by sophisticated cellular machinery that blocks $60 \mathrm{~S}$ exporting to the cytoplasm if its integrity is compromised ${ }^{22}$. No clear function has been assigned for this loop that would justify such a conserved and costly cellular machinery ${ }^{23}$.

Here, residues Ser104 to Arg110 of this loop are located in close proximity to the ${ }_{73} \mathrm{ACCA}_{76}$-Met of Met-tRNA $\mathrm{A}_{\mathrm{i}} \mathrm{Met}$, in an almost parallel configuration to the phosphate backbone of the Met-tRNA $A_{i}$ Met (Fig. 3c). In addition, uL16 residues Leu103 and Ser104 tightly approach the eIF5B residues around Leu871 to define a narrow and hydrophobic cavity where the methionine residue is hosted (Fig. 3d and Supplementary Fig. 6). No specific 

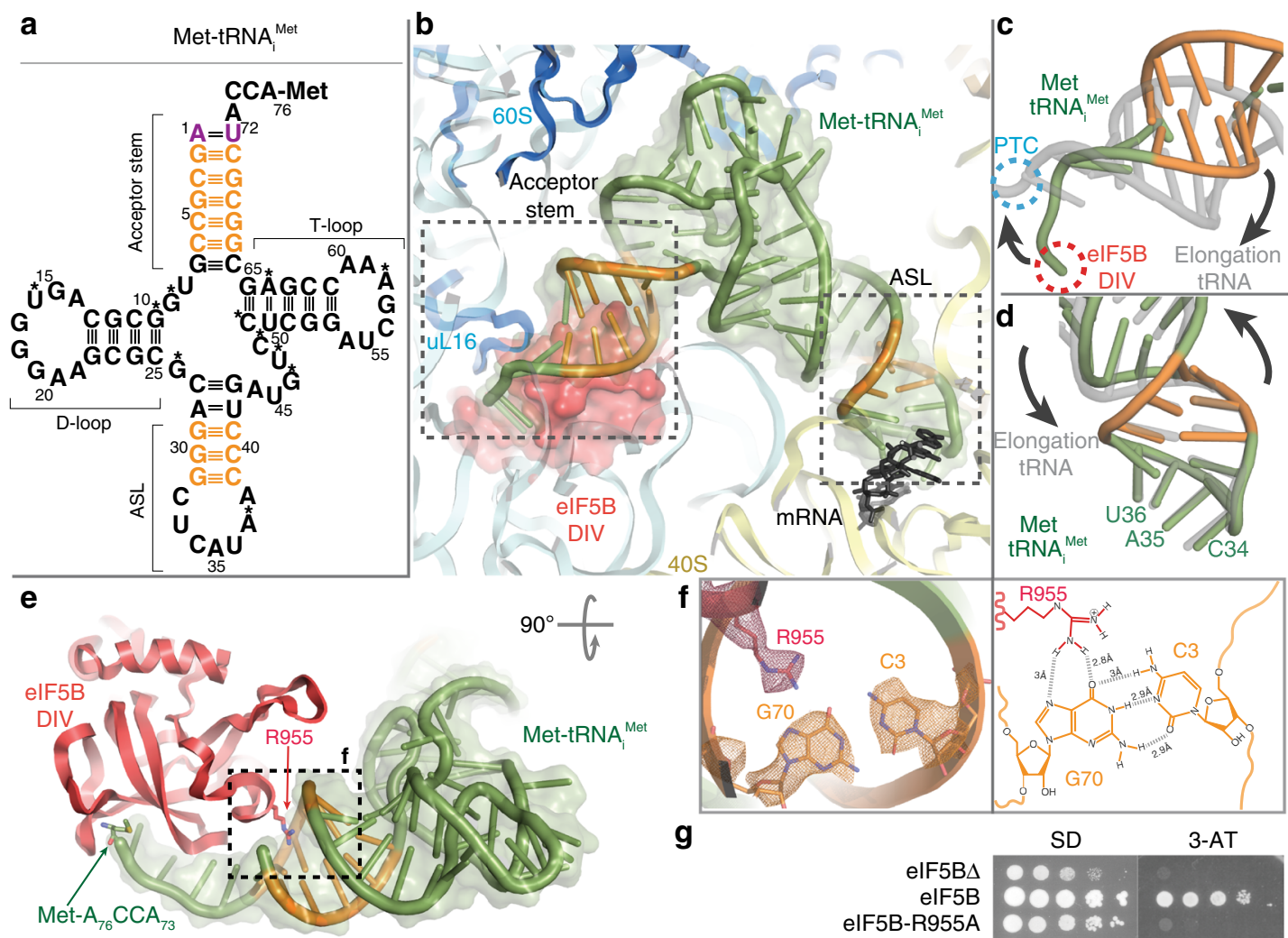

R955

$$
\text { g }
$$
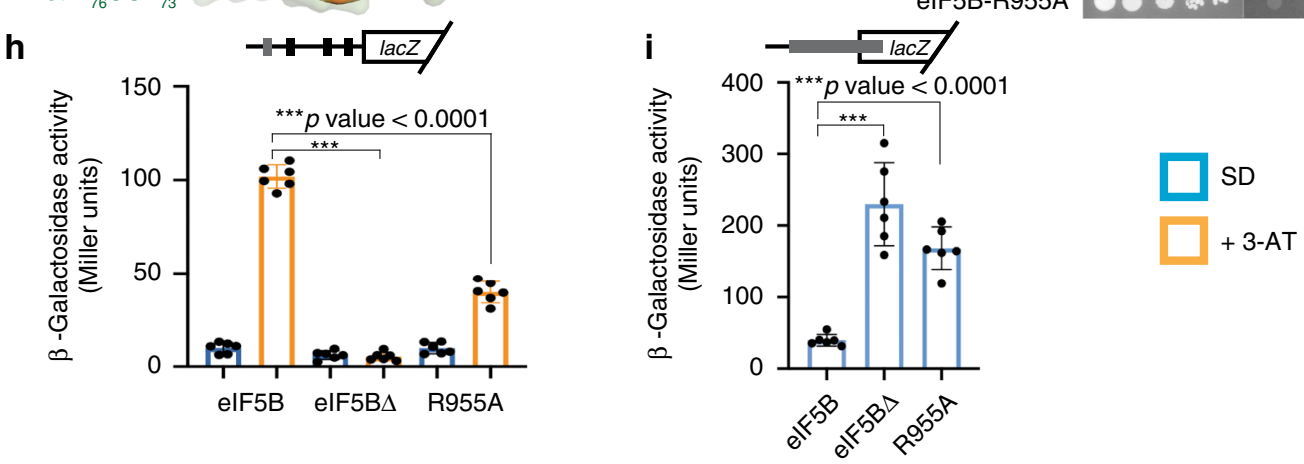

Fig. 2 Role of the G-C base-pairs clusters in Met-tRNA $\mathbf{A}_{\mathbf{i}}^{\text {Met }}$ conformation. a Sequence diagram of the S. cerevisiae Met-tRNA $A_{i}$ Met with initiator-specific base pairs colored in orange and modified nucleotides marked with asterisks. $\mathbf{b}$ Overview of the conformation adopted by Met-tRNA ${ }_{i}{ }^{\text {Met }}$ on the $80 S-I C$ complex with the initiator-specific G-C base pairs colored in orange. c Superposition of Met-tRNA $A_{i}$ Met (green) with a P site classical-state elongation tRNA (gray) reveals a distorted configuration of the acceptor stem of Met-tRNA $A_{i}$ Met when compared with an elongation peptidyl-tRNA. This distortion prevents the ${ }_{74} \mathrm{CCA}_{76}$-Met end of Met-tRNA ${ }_{i}$ Met from reaching the peptidyl transferase center (PTC, blue) on the $60 \mathrm{~S}$. $\mathbf{d}$ In contrast, the ASL of Met-tRNA ${ }_{i}$ Met has reached its final position when compared with an elongation tRNA in the P site. e The arginine residue 955 at DIV of elF5B specifically recognizes the Hoogsteen edge of the base of nucleotide G70 of Met-tRNA ${ }_{i}$ Met (red arrow, R955). $\mathbf{f}$ Cryo-EM density for side chains and individual bases in the area around elF5B R955. On the right, chemical diagram with contacts and distances indicated. g-i Yeast growth and GCN4-lacZ reporter assays of strains lacking elF5B or expressing wild-type or R955A mutant. Cells were spotted on minimal (SD) or starvation (3-AT) medium (g) or assayed for Bgalactosidase activity following introduction of a wild-type GCN4-laZ reporter (h) or a derivative in which an extended version of uORF1 overlaps, out-offrame, the GCN4 start codon (i). Results are the average and standard deviation of six independent transformants combined from two independent experiments with three replicates each; $p$ values were calculated using two-sided Student's $t$ test. The $p$ values for panel (h) are elF5B vs. elF5B $\Delta$ : $7.57349 \times 10^{-12}$, elF5B vs. elF5B-R955A: $6.30158 \times 10^{-9}$ and for panel (i) are elF5B vs. elF5BA: $1.23215 \times 10^{-5}$, elF5B vs. elF5B-R955A: 1.3313 × 10-6.

contacts between the methionine moiety and either eIF5B or uL16 residues could be observed, which suggests that the recognition of the amino acid attached to an initiator tRNA is not achieved via a specific interaction with eIF5B or uL16 residues. To explore the determinants that govern such recognition mechanism, we systematically mis-acylated $\mathrm{tRNA}_{\mathrm{i}} \mathrm{Met}^{\mathrm{mith}}$ five different amino acids with increasing side chain volumes and hydrophobicity or introducing a positive charge (Fig. 4$)^{24}$.

All the mis-acylated tRNAs are active in 48S PIC and $80 \mathrm{~S}$ formation (Fig. 4). However, the $60 \mathrm{~S}$ subunit joining rate is altered in a way that reflects a predilection for larger side chains: the stepwise increase of the side chain size from Gly to Ala, Val, Leu, and even the positively charged Lys resulted in increasing $60 \mathrm{~S}$ joining rates, progressively approaching the values observed for Met (Fig. 4a, c). In addition, the mean times for the transition to elongation all decreased substantially to a similar level (by $\sim 3-5$-fold) due to the mis-acylation. Both the altered $60 \mathrm{~S}$ arrival times and faster transitions into elongation might reflect weakened interactions between the aminoacyl-tRNA $\mathrm{A}_{\mathrm{i}}$ Met and eIF5B/uL16 when the methionine moiety was replaced by other 

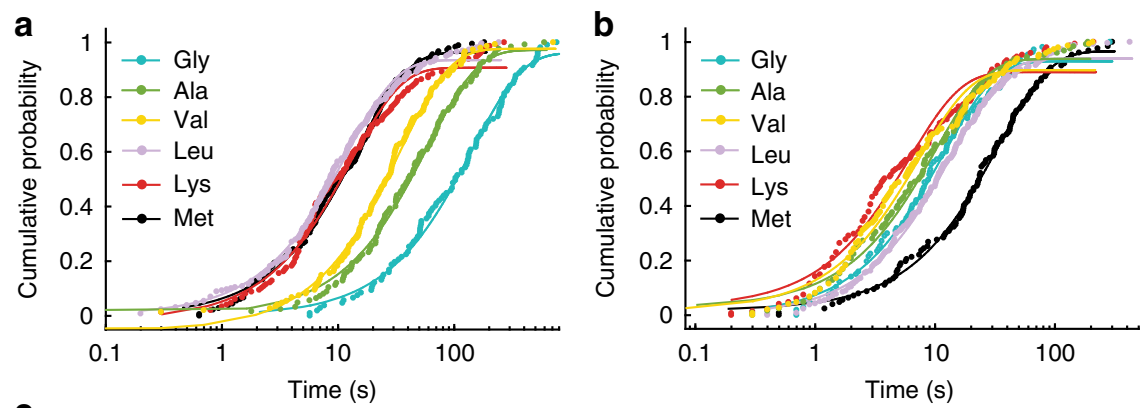

\begin{tabular}{cccccc} 
C & \multicolumn{3}{c}{} & & \\
\hline $\begin{array}{c}\text { Aminoacyl- } \\
\text { tRNA }_{i}^{\text {Met }}\end{array}$ & $\begin{array}{c}\text { Time for 60S } \\
\text { joining(s) }\end{array}$ & Fold change & $\begin{array}{c}\text { Time for the transition } \\
\text { to elongation }(\mathrm{s})\end{array}$ & Fold change & $\begin{array}{c}\text { Number of } \\
\text { molecules }(n)\end{array}$ \\
\hline Gly-tRNA $_{\mathrm{i}}^{\text {Met }}$ & $142.9 \pm 6.2$ & 9.7 & $11.7 \pm 0.5$ & 0.3 & 107 \\
Ala-tRNA $_{\mathrm{i}}^{\text {Met }}$ & $58.6 \pm 1.4$ & 4.0 & $10.1 \pm 0.5$ & 0.3 & 125 \\
Val-tRNA $_{\mathrm{i}}^{\text {Met }}$ & $33.9 \pm 0.9$ & 2.3 & $7.4 \pm 0.6$ & 0.2 & 148 \\
Leu-tRNA $_{\mathrm{i}}^{\text {Met }}$ & $11.9 \pm 0.3$ & 0.8 & $14.1 \pm 0.3$ & 0.4 & 259 \\
Lys-tRNA $_{\mathrm{i}}^{\text {Met }}$ & $13 \pm 0.7$ & 0.9 & $6.1 \pm 0.6$ & 0.2 & 148 \\
Met-tRNA $_{\mathrm{i}}^{\text {Met }}$ & $14.8 \pm 0.4$ & 1 & $33.7 \pm 0.9$ & 1 & 127 \\
\hline
\end{tabular}

Fig. 4 Kinetics of $60 \mathrm{~S}$ subunit joining and the subsequent transition into elongation in experiments performed with mis-acylated tRNA $\mathrm{A}_{i}$ et. $^{\text {The }}$ cumulative probability distributions of the dwell times for $60 \mathrm{~S}$ joining (a) and the transition to elongation (b) from experiments performed at $3 \mathrm{mM}$ free $\mathrm{Mg}^{2+}$ and $20^{\circ} \mathrm{C}$ with the model mRNA and unlabeled elF5B. Data points were fitted to a single-exponential equation with the mean times reported in (c). Errors represent $95 \%$ confidence intervals, and the number of molecules analyzed $(n)$ are listed in (c).

amino acids, reflecting the importance of the size of the side chain esterified to A76 of tRNA ${ }_{i}{ }^{\text {Met }}$. The cavity formed by eIF5B DIV and the uL16 loop (residues 100-120) could thus act as a selectivity "filter" regarding the identity of the amino acid.

AUG recognition by the Met-tRNA $\mathrm{A}_{\mathbf{i}}$ Met anticodon. The anticodon arm of Met-tRNA ${ }_{i}{ }^{\text {Met }}$ features a configuration very similar to that described for a peptidyl-tRNA in an elongation, canonical state (Fig. 5, Supplementary Fig. 5, and Supplementary Movie 1) ${ }^{20}$. Anticodon bases C34, A35, and U36 have reached their final elongation position, which would allow a productive transition into elongation. The G-C cluster of base pairs in the ASL seems to play an important role, allowing a local distortion that guarantees an ideal positioning of the anticodon bases to maximize the interaction with the AUG codon ${ }^{15}$, while allowing the tRNA to bend at the elbow region of T-loop/D-loop tertiary interaction to prevent accommodation of the aminoacyl-acceptor stem to the elongation state (Fig. $2 \mathrm{~b}-\mathrm{d}$ ). In addition, the A minor type interactions between $40 \mathrm{~S}$ residues at the $\mathrm{P}$ site and the MettRNA $_{i}{ }^{M e t} \mathrm{ASL}$ at the G-C base-pair cluster are similar to those described to be important in bacterial initiation, highlighting the functional importance of the G-C base-pair cluster in the ASL of tRNA $_{i}{ }^{\text {Met }}$ throughout evolution (Supplementary Fig. 5c) ${ }^{25}$. Thus, both $\mathrm{G}-\mathrm{C}$ base-pair clusters at the acceptor stem and the anticodon arm of Met-tRNA ${ }_{i}$ Met are essential to allow a specific conformation of the Met-tRNA $\mathrm{A}_{\mathrm{i}}$ Met on the $\mathrm{P}$ site in both the early $^{21}$ and late stages of initiation ${ }^{14}$.

For the mRNA, we could unambiguously identify six nucleotides including the AUG start codon and the three nucleotides immediately upstream (Fig. 5). Only weak densities ascribable to the A-site codon could be observed, and no ordered RNA density could be identified at the entry nor exit sites of the mRNA channel on the 40S. This is in marked contrast with $48 \mathrm{~S}$ PIC structures from earlier initiation stages, in which long stretches of the mRNA from the entry to the exit sites were well resolved $^{5}$, pinpointing a less prominent role of $\mathrm{mRNA} / 40 \mathrm{~S}$ interactions once initiation has progressed into its later stages.

The codon-anticodon interaction observed in our reconstruction is virtually identical to a canonical cognate pair (Fig. $5 b, c)^{20}$. Nucleotides C1637 and U1191 of the 18S rRNA at the base of the
40S P-site bracket the base pair mRNA- ${ }_{3} \mathrm{G} \mathrm{C}_{34}-\mathrm{tRNA}_{\mathrm{i}}{ }^{\text {Met }}$ in a very similar configuration as in an elongation complex (Fig. 5c, d). In addition, ribosomal bases G1150 and G904 engage mRNA nucleotides at position -1 and -3 , respectively, in stacking interactions in a similar configuration as in early initiation complexes in the 48 S PIC context (Fig. $5 c, d)^{6,7}$. Thus, in the later stages of initiation, prior to entrance into elongation, the start codon and its flanking residues present a hybrid configuration with bases at position $-1 /-3$ retaining key contacts with rRNA bases that are instrumental in the early positioning of the mRNA on the $40 \mathrm{~S}$ and, at the same time, with the start-codon features characteristic of a standard conformation of an elongation state ${ }^{20}$. Hence, the start codon-surrounding sequence, especially the bases at positions $-3 /-1$, plays an essential role along all initiation, from early "scanning" to later entrance into elongation ${ }^{26}$.

Release of eIF5B-GDP limits the rate of the transition into elongation. Analysis of the G domain of eIF5B that binds GTP reveals clear density for a bound nucleotide that, however, lacks features compatible with the presence of a $\gamma$-phosphate, implying that the nucleotide state is either GDP or GDP-Pi (Supplementary Fig. $4 \mathrm{~d})^{27,28}$. The absence of density ascribable to the $\gamma$ phosphate even at a low threshold (Supplementary Fig. 4d, right) points towards a nucleotide state with GDP. This is additionally supported by the fact that the switch I of eIF5B is disordered, which differs from the ordered state prior to GTP hydrolysis ${ }^{27,29}$. Thus, our reconstruction represents an intermediate of the 80S IC right before its transition to the elongation-competent state, but post GTP hydrolysis. This is further supported by the fact that the ribosomal inter-subunit configuration observed here is different from the 80S IC state prior to GTP hydrolysis (Supplementary Fig. 7). $\mathrm{A} \sim 3^{\circ}$ counterclockwise rotation of the $40 \mathrm{~S}$ in relation to the $60 \mathrm{~S}$ was observed in the pre-GTP hydrolysis state, which was coupled with apparent 40S head swivel (Supplementary Fig. $7^{12,13,30}$ ). However, the eIF5B-bound 80 S IC observed here presents a configuration very similar to a canonical non-rotated $80 S$ complex, with virtually no rotation of the small subunit and minimal swiveling of the $40 \mathrm{~S}$ head (Supplementary Fig. 7). Thus, the 80S IC will reconfigure its conformation after GTP hydrolysis to a state that is more similar to the non-rotated 


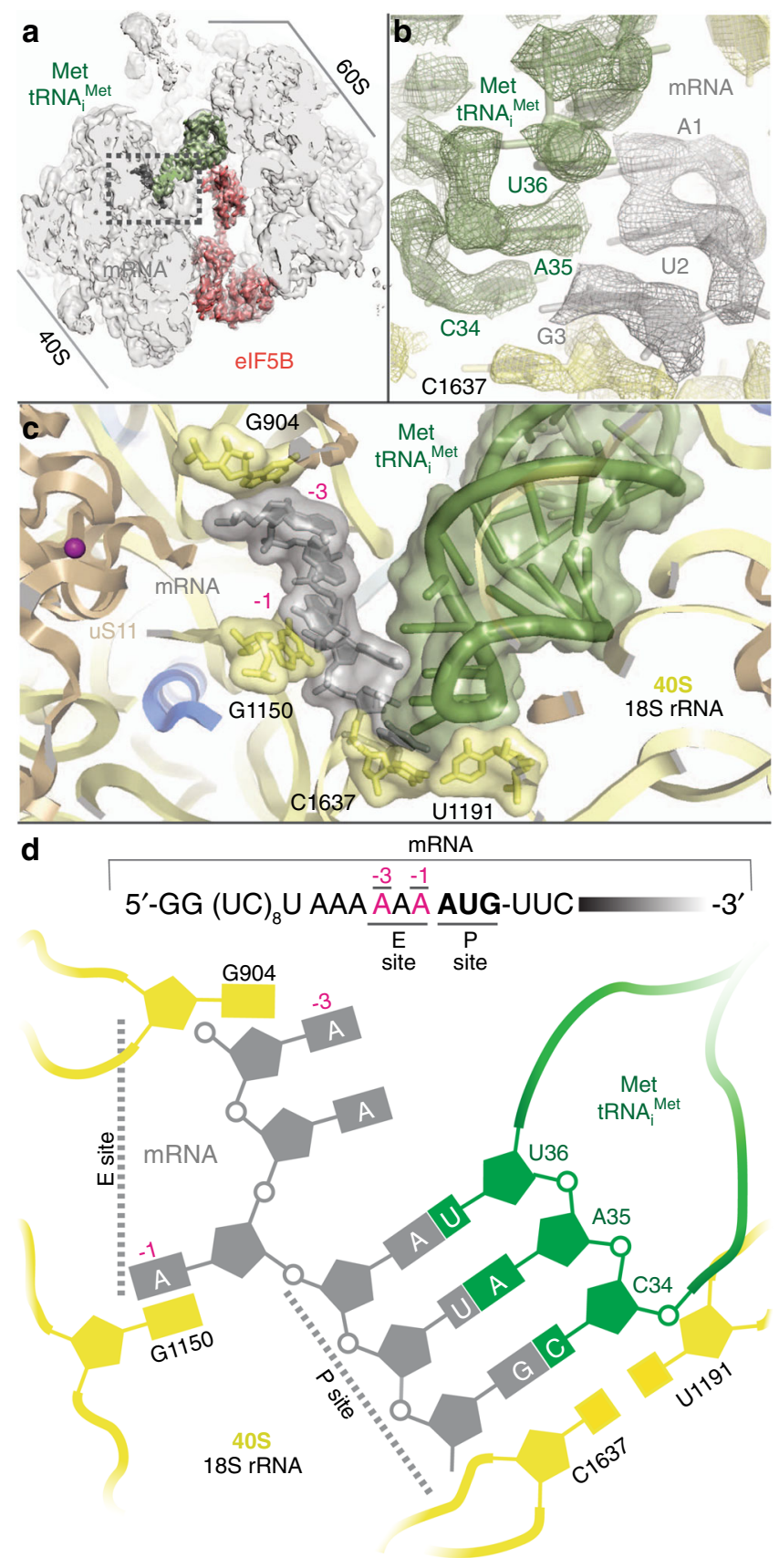

Fig. 5 mRNA nucleotides at positions $-1 /-3$ play a key role in later stages of initiation. a Overall view of the $80 \mathrm{~S} /$ Met-tRNA $_{i}$ Met/elF5B complex in an orientation centered on the mRNA. b Detailed view of the small ribosomal subunit $P$ site with experimental cryo-EM density shown. The Met-tRNA $A_{i}$ Met ASL is in green, the start codon gray, and ribosomal bases of the small subunit yellow. c Overall view of the P and E sites of the small ribosomal subunit. Six bases corresponding to the start codon of the mRNA and three preceding bases corresponding to nucleotides -1 to -3 showed unambiguous densities and could be modeled (gray). The final refined model with labeled components is shown. $\mathbf{d}$ Simplified schematic view of the conformation adopted by the start codon in the $P$ site and the preceding -1 to -3 bases in the $E$ site in the presence of elF5B.

elongation-competent state, and this reconfiguration is coupled to the structural rearrangements of eIF5B.

In summary, our structural analysis of the pre-steady-state initiation complexes identified a novel intermediate of the late translation initiation complex on the native reaction pathway.
Our results demonstrated that GTP hydrolysis and Pi release are not the rate-limiting steps for eIF5B dissociation from the 80S IC, but the subsequent eIF5B-GDP dissociation. Notably, our structure describes stable interactions among Met-tRNA $\mathrm{Met}_{\mathrm{i}}$, eIF5B DIV, and uL16 after GTP hydrolysis by eIF5B (Fig. 6). Disruption of this network of contacts is required for release of eIF5B, perhaps explaining the slow eIF5B departure rate post GTP hydrolysis. Such cooperation among Met-tRNA $\mathrm{A}_{\mathrm{i}}$, an initiation factor and the 60S subunit highlights a eukaryotespecific mechanism to control the progression of the initiation complex into the elongation phase.

\section{Methods \\ Materials. All the yeast Saccharomyces cerevisiae 40S and 60S ribosomal subunits, initiation factors eIF1, 1A, 2, 5, and 5B (residues 396-1002), and mRNA were prepared and characterized as described ${ }^{11}$. The model mRNA, with the sequence: $\mathrm{GG}(\mathrm{UC})_{8} \mathrm{UAAAAAAAUGUUCAAAUAA}(\mathrm{UC})_{16}$, was an uncapped, unstructured model mRNA containing the optimal yeast $5^{\prime}$ context consensus sequence (underlined) with a biotin covalently linked to the $3^{\prime} \mathrm{end}^{11}$. Native yeast methio- nylated initiator tRNA (Met-tRNA $\mathrm{i}_{\mathrm{i}}^{\text {Met }}$ ) was purchased from tRNA Probes, LLC (MI-60).}

80S:elF5B complex assembly on model mRNA. Previously, by applying singlemolecule fluorescence microscopy methods to a purified, reconstituted yeast translation system, we have revealed that eIF5B is the gating factor during the transition from eukaryotic translation initiation to elongation ${ }^{11}$. We preformed post-scanning $48 \mathrm{~S}$ preinitiation complexes (48S PICs, wherein $40 \mathrm{~S}$ was labeled with a Cy3 dye) which were immobilized on zero-mode waveguides (ZMWs) imaging surface via a biotin at the $3^{\prime}$ end of the mRNAs (Supplementary Fig. 1a) ${ }^{11}$. After washing away unbound components, 60S (Cy5-labeled), eIF5B (Cy5.5-labeled), and the first elongator Phe-tRNA $A^{\text {Phe }}$ (Cy3.5-labeled) in ternary complex with the elongation factor eEF1A and GTP (Phe-TC) were delivered along with required eIFs to start the reaction. By directly illuminating all the fluorescent dyes, we could monitor, in real time, the order of molecular events occurring during the late translation initiation stages and its transition to elongation (Supplementary Fig. 1b) ${ }^{11}$. This allowed us to measure and determine the occupancy times of eIF5B on newly formed $80 \mathrm{~S}$ complexes on the native reaction pathway. In particular, for the model mRNA, the mean time of $60 \mathrm{~S}$ joining to form an $80 \mathrm{~S}$ was $\sim 16 \mathrm{~s}$ with the eIF5B occupancy time on the $80 \mathrm{~S} \sim 34 \mathrm{~s}$ at $20^{\circ} \mathrm{C}$ and $3 \mathrm{mM}$ free $\mathrm{Mg}^{2+}$; whereas these values were $\sim 3.6 \mathrm{~s}$ and $68.7 \mathrm{~s}$ at $20^{\circ} \mathrm{C}$ and $10 \mathrm{mM}$ free $\mathrm{Mg}^{2+}$, respectively ${ }^{11}$. Simulating the kinetic curves from these two reactions provided the information about the time-evolution of the populations of different complexes (Supplementary Fig. 1c) ${ }^{11}$. To aid the reconstruction of a high-resolution structure of the onpathway eIF5B-bound $80 \mathrm{~S}$ prior to its transition to elongation without the need of non-hydrolysable GTP analogs or mutants, we decided to prepare and freeze our sample at timepoint $40 \mathrm{~s}$ at $20^{\circ} \mathrm{C}$ and $10 \mathrm{mM}$ free $\mathrm{Mg}^{2+}$, where we would expect the eIF5B-bound 80 S population accounts $\sim 60 \%$ of the total 80 S particles (Supplementary Fig. 1c).

Samples were prepared in the buffer containing $30 \mathrm{mM}$ HEPES-KOH pH 7.5, $100 \mathrm{mM} \mathrm{KOAc}, 10 \mathrm{mM} \mathrm{Mg}(\mathrm{OAc})_{2}$, and $1 \mathrm{mM} \mathrm{GTP}: \mathrm{Mg}^{2+}$. First, a ternary complex mixture was prepared by pre-incubating $3.8 \mu \mathrm{M}$ eIF2 at $30^{\circ} \mathrm{C}$ for $10 \mathrm{~min}$, followed by another $5 \mathrm{~min}$ incubation at $30^{\circ} \mathrm{C}$ after addition of $2.8 \mu \mathrm{M}$ Met-tRNA $\mathrm{i}_{\mathrm{i}}$. Next, this ternary complex mixture was diluted to one-third of the concentration, and incubated at $30^{\circ} \mathrm{C}$ together with $1 \mu \mathrm{M}$ eIF1, $1 \mu \mathrm{M}$ eIF1A, $0.5 \mu \mathrm{M}$ model mRNA, and $0.3 \mu \mathrm{M} 40 \mathrm{~S}$ for $15 \mathrm{~min}$, resulting a 48S PIC mixture. Separately, a 60S mixture was prepared by mixing $1 \mu \mathrm{M}$ eIF5, $1 \mu \mathrm{M}$ eIF5B, and $0.15 \mu \mathrm{M}$ 60S. The 48S PIC and 60S mixtures were kept on ice before sample freezing. In parallel, the 200-mesh Quantifoil R2/1 grids (Electron Microscopy Sciences, Q250AR1) were glowdischarged for $25 \mathrm{~s}$ in a PELCO EasiGlow glow discharger (Ted Pella, Inc., conditions: negative charge, $15 \mathrm{~mA}, 0.4 \mathrm{mBar}, 25 \mathrm{~s}$ ). After prewarming the samples to room temperature, $3.5 \mu \mathrm{L}$ of the $48 \mathrm{~S}$ PIC mixture was mixed with $3.5 \mu \mathrm{L}$ of the $60 \mathrm{~S}$ mixture. A $3 \mu \mathrm{L}$ sample from the resulting mixture was applied to each grid at $21^{\circ} \mathrm{C}$ and $95 \%$ humidity. The sample was vitrified by plunging into liquid ethane after $2.5 \mathrm{~s}$ blotting using a Leica EM GP (Leica Microsystems) plunger. The total time from combining the $48 \mathrm{~S}$ PIC and $60 \mathrm{~S}$ mixtures to grid freezing was $\sim 40 \mathrm{~s}$.

Single-molecule experiments with mis-acylated tRNA $\mathbf{A}_{i}$ Met. Native yeast $\mathrm{tRNA}_{\mathrm{i}} \mathrm{Met}^{\mathrm{M}}$ was mis-acylated by the flexizyme dFx with Gly-, Ala-, Val-, Leu-, and Lys-DBE as described ${ }^{24,31}$. Real-time single-molecule experiments on the ZMWbased PacBio RSII instrumentation and data analyses were performed with exactly the same methodology as previously described ${ }^{11}$. The mean times of $60 \mathrm{~S}$ arrival to the immobilized 48 S PICs (60S arrival time) and the subsequent transition to elongation were determined in experiments performed with the model mRNA and unlabeled eIF5B at $3 \mathrm{mM}$ free $\mathrm{Mg}^{2+}$ and $20^{\circ} \mathrm{C}$. 

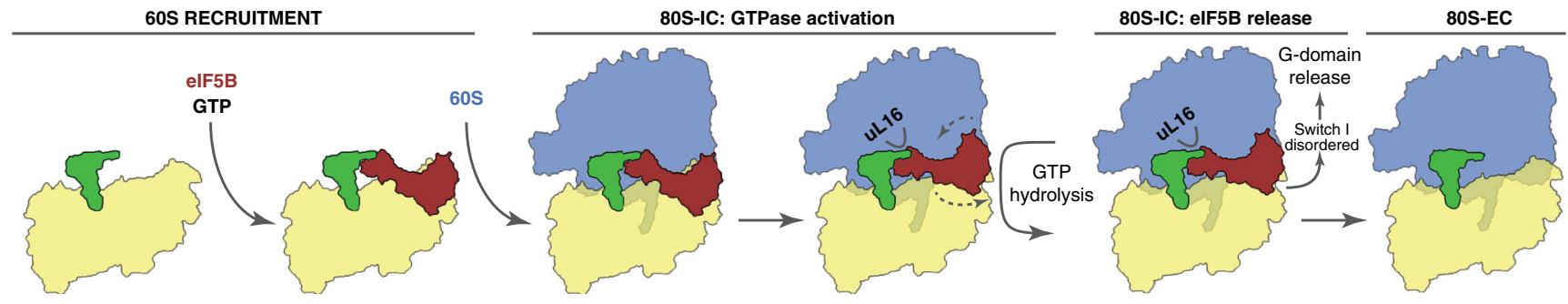

Fig. 6 A structural view of the on-ribosome elF5B catalysis. After Met-tRNA $A_{i}$ Met (green) is delivered to the P site of the small subunit (4OS, yellow) and the start codon is recognized, elF5B in its GTP-bound form is able to transiently stabilize the aminoacyl-tRNA in the P site and catalyze the recruitment of the large subunit (60S, blue). Proper positioning of Met-tRNA $A_{i}$ Met, elF5B, and the 60S would lead to the structuring of the uL16 loop (residues 100-120) which in turn anchors elF5B DIV solidly to the $60 \mathrm{~S}$ in the vicinity of the PTC. The successful establishment of these interactions signals for elF5B GTPase activation and GTP hydrolysis, which happens in the G domain of elF5B placed near the GTPase activation center of the 60S. GTP hydrolysis by elF5B is followed by the de-structuring of switch I, which reduces the affinity of the factor to the ribosome. The final departure of elF5B from the ribosome is likely implemented in two steps, beginning with the super-domain G/II and followed by the detachment of DIV-h12-DIII.

Generation of elF5B mutant and growth and reporter experiments in yeast. The Saccharomyces cerevisiae fun124 strain J111 (MATa ura3-52 leu2-3 leu2-112 fun12 $\Delta)^{32}$ lacking eIF5B was transformed with an empty vector (eIF5B $\Delta$ ) or plasmids expressing $\mathrm{N}$-terminally deleted (lacking residues 28-396) form of wild-type (WT) eIF5B 32 or the eIF5B-R955A mutant, as indicated. Transformants were grown to saturation, and $5 \mu \mathrm{l}$ of serial dilutions $\left(\right.$ of $\mathrm{OD}_{600 \mathrm{~nm}}=1.0,0.1,0.01$, 0.001 , and 0.0001 ) were spotted on minimal medium supplemented with essential nutrients (SD) or medium containing $10 \mathrm{mM} \mathrm{3-aminotriazole} \mathrm{(3-AT)} \mathrm{to} \mathrm{cause}$ histidine starvation. Plates were incubated for 3 days at $30^{\circ} \mathrm{C}$. For reporter assays, wild-type GCN4-lacZ plasmid $180^{33}$ or a deletion derivative in which uORF1 was moved to the position of uORF4 and then extended to overlap the GCN4 AUG start codon (pM226) ${ }^{34}$ were introduced into the strains. Cells were grown under repressing, non-starvation, conditions in SD medium for $2 \mathrm{~h}$, and then incubated under repressing or derepressing, starvation, conditions (SD + $10 \mathrm{mM} 3-\mathrm{AT}$ ) for an additional $6 \mathrm{~h}$; cells were harvested and B-galactosidase activities were determined as described previously ${ }^{34,35}$.

Data collection, image processing, and structure determination. Cryo-EM data was collected on a Titan Krios microscope (FEI) equipped with an energy filter (20 $\mathrm{eV}$ slit width) and a $\mathrm{K} 3$ direct detector (Gatan) operated at $300 \mathrm{KeV}$. A $70 \mu \mathrm{m} \mathrm{C} 2$ aperture was used with a pixel size of $0.83 \AA$ A pixel and illumination conditions adjusted in nanoprobe mode to a fluence of $10 \mathrm{e}^{-} / \mathrm{pixel} / \mathrm{s}$. Four-second images with a frame width of $100 \mathrm{~ms}\left(1.45 \mathrm{e}^{-} / \AA^{2} /\right.$ frame $)$ were collected in counting mode. Contrast transfer function parameters were estimated using CTFIND4 ${ }^{36}$. Particle picking was performed using Relion $3.1^{18}$ without the use of templates and with the Laplacian module ( $\max / \mathrm{min}$ diameter $380 / 320 \AA$ ) identifying an initial set of particles. Eight times binned particles (extraction box 416 pixels) were subjected to an initial 2D classification job (200 classes, T parameter 2, 35 iterations) and those classes showing clear secondary structure features were selected for a 8xbin 3D auto-refine job. All 2D and 3D classifications and refinements were performed using RELION ${ }^{17,18,37}$

Next, 3D classification without alignment and a mask including the intersubunit space and the $40 \mathrm{~S}$ head (four classes, $\mathrm{T}$ parameter 4 ) identified a class with unambiguous density for eIF5B and a tRNA in the P site ${ }^{17}$. This class was independently processed with unbinned data, yielding high-resolution maps with density features in agreement with the reported resolution after several cycles of ctf-refinement and a final step of Bayesian polishing. Local resolution was computed with RESMAP ${ }^{38}$.

Model building and refinement. Models of yeast $40 \mathrm{~S}, 60 \mathrm{~S}^{27}, \mathrm{Met}-\mathrm{tRNA} \mathrm{A}_{\mathrm{i}}^{\mathrm{Met}}$, and eIF5B were docked into the maps using CHIMERA ${ }^{39}$, and COOT $^{40}$ was used to manually adjust these initial models. An initial round of refinement was performed in Phenix using real-space refinement ${ }^{41}$ with secondary structure restraints and a final step of reciprocal-space refinement with REFMAC ${ }^{42}$. The fit of the model to the map over-fitting tests were performed following standard protocols in the field ${ }^{43}$.

Reporting summary. Further information on research design is available in the Nature Research Reporting Summary linked to this article.

\section{Data availability}

The data that support this study are available from the corresponding authors upon reasonable request. Cryo-EM maps have been deposited in the Electron Microscopy Data Bank under accession code EMD-21859 and coordinates at the Protein Data Bank under accession code 6WOO. Source data for Fig. $2 \mathrm{~h}$, i are included as Supplementary Table 2 .

\section{Code availability}

The MATLAB scripts used for single-molecule data analyses are available at https://drive. google.com/drive/u/1/folders/1-yKC0Nui7T7yoJScCuODPhCT4G52emLj.

Received: 13 June 2020; Accepted: 16 September 2020; Published online: 06 October 2020

\section{References}

1. Frank, J. \& Gonzalez, R. L. Jr. Structure and dynamics of a processive Brownian motor: the translating ribosome. Annu Rev. Biochem 79, 381-412 (2010).

2. Aitken, C. E. \& Lorsch, J. R. A mechanistic overview of translation initiation in eukaryotes. Nat. Struct. Mol. Biol. 19, 568-576 (2012).

3. Jackson, R. J., Hellen, C. U. \& Pestova, T. V. The mechanism of eukaryotic translation initiation and principles of its regulation. Nat. Rev. Mol. Cell Biol. 11, 113-127 (2010).

4. Hinnebusch, A. G. The scanning mechanism of eukaryotic translation initiation. Annu Rev. Biochem 83, 779-812 (2014).

5. Hinnebusch, A. G. Structural Insights into the Mechanism of Scanning and Start Codon Recognition in Eukaryotic Translation Initiation. Trends Biochem Sci. 42, 589-611 (2017).

6. Llacer, J. L. et al. Translational initiation factor eIF5 replaces eIF1 on the 40S ribosomal subunit to promote start-codon recognition. Elife 7, https://doi.org/ 10.7554/eLife.39273 (2018).

7. Hussain, T. et al. Structural changes enable start codon recognition by the eukaryotic translation initiation complex. Cell 159, 597-607 (2014).

8. Hashem, Y. \& Frank, J. The jigsaw puzzle of mRNA translation initiation in eukaryotes: a decade of structures unraveling the mechanics of the process. Annu Rev. Biophys. https://doi.org/10.1146/annurev-biophys-070816-034034 (2018).

9. Pestova, T. V. et al. The joining of ribosomal subunits in eukaryotes requires eIF5B. Nature 403, 332-335 (2000).

10. Dever, T. E. et al. Universal translation initiation factor IF2/eIF5B. Cold Spring Harb. Symp. Quant. Biol. 66, 417-424 (2001).

11. Wang, J. et al. eIF5B gates the transition from translation initiation to elongation. Nature 573, 605-608 (2019).

12. Fernandez, I. S. et al. Molecular architecture of a eukaryotic translational initiation complex. Science 342, 1240585 (2013).

13. Yamamoto, H. et al. Structure of the mammalian $80 \mathrm{~S}$ initiation complex with initiation factor 5B on HCV-IRES RNA. Nat. Struct. Mol. Biol. 21, 721-727 (2014).

14. Kolitz, S. E. \& Lorsch, J. R. Eukaryotic initiator tRNA: finely tuned and ready for action. FEBS Lett. 584, 396-404 (2010).

15. Kolitz, S. E., Takacs, J. E. \& Lorsch, J. R. Kinetic and thermodynamic analysis of the role of start codon/anticodon base pairing during eukaryotic translation initiation. RNA 15, 138-152 (2009).

16. Acker, M. G. et al. Kinetic analysis of late steps of eukaryotic translation initiation. J. Mol. Biol. 385, 491-506 (2009).

17. Scheres, S. H. Processing of structurally heterogeneous Cryo-EM Data in RELION. Methods Enzymol. 579, 125-157 (2016).

18. Zivanov, J. et al. New tools for automated high-resolution cryo-EM structure determination in RELION-3. Elife 7, https://doi.org/10.7554/eLife.42166 (2018). 
19. Shin, B. S. et al. Structural integrity of \{alpha\}-helix H12 in translation initiation factor eIF5B is critical for 80S complex stability. RNA 17, 687-696 (2011).

20. Shao, S. et al. Decoding mammalian ribosome-mRNA states by translational GTPase complexes. Cell 167, 1229-1240 e1215 (2016).

21. Dong, J. et al. Conserved residues in yeast initiator tRNA calibrate initiation accuracy by regulating preinitiation complex stability at the start codon. Genes Dev. 28, 502-520 (2014).

22. Sulima, S. O. et al. Bypass of the pre-60S ribosomal quality control as a pathway to oncogenesis. Proc. Natl Acad. Sci. USA 111, 5640-5645 (2014).

23. Sulima, S. O. et al. Eukaryotic rpL10 drives ribosomal rotation. Nucleic Acids Res. 42, 2049-2063 (2014).

24. Murakami, H., Ohta, A., Ashigai, H. \& Suga, H. A highly flexible tRNA acylation method for non-natural polypeptide synthesis. Nat. Methods 3, 357-359 (2006).

25. Lancaster, L. \& Noller, H. F. Involvement of 16S rRNA nucleotides G1338 and A1339 in discrimination of initiator tRNA. Mol. Cell 20, 623-632 (2005).

26. Kozak, M. The scanning model for translation: an update. J. Cell Biol. 108, 229-241 (1989).

27. Huang, B. Y. \& Fernandez, I. S. Long-range interdomain communications in eIF5B regulate GTP hydrolysis and translation initiation. Proc. Natl Acad. Sci. USA 117, 1429-1437 (2020).

28. Kuhle, B. \& Ficner, R. eIF5B employs a novel domain release mechanism to catalyze ribosomal subunit joining. EMBO J. 33, 1177-1191 (2014).

29. Shin, B. S. et al. rRNA suppressor of a eukaryotic translation initiation factor 5B/initiation factor 2 mutant reveals a binding site for translational GTPases on the small ribosomal subunit. Mol. Cell Biol. 29, 808-821 (2009).

30. Sprink, T. et al. Structures of ribosome-bound initiation factor 2 reveal the mechanism of subunit association. Sci. Adv. 2, e1501502 (2016).

31. Peacock, J. R. et al. Amino acid-dependent stability of the acyl linkage in aminoacyl-tRNA. RNA 20, 758-764 (2014)

32. Shin, B. S. et al. Intragenic suppressor mutations restore GTPase and translation functions of a eukaryotic initiation factor 5B switch II mutant. Mol. Cell Biol. 27, 1677-1685 (2007).

33. Hinnebusch, A. G. A hierarchy of trans-acting factors modulates translation of an activator of amino acid biosynthetic genes in Saccharomyces cerevisiae. Mol. Cell Biol. 5, 2349-2360 (1985).

34. Grant, C. M., Miller, P. F. \& Hinnebusch, A. G. Requirements for intercistronic distance and level of eukaryotic initiation factor 2 activity in reinitiation on GCN4 mRNA vary with the downstream cistron. Mol. Cell Biol. 14, 2616-2628 (1994).

35. Shin, B. S. et al. Uncoupling of initiation factor eIF5B/IF2 GTPase and translational activities by mutations that lower ribosome affinity. Cell 111, 1015-1025 (2002).

36. Rohou, A. \& Grigorieff, N. CTFFIND4: fast and accurate defocus estimation from electron micrographs. J. Struct. Biol. 192, 216-221 (2015).

37. Zivanov, J., Nakane, T. \& Scheres, S. H. W. Estimation of high-order aberrations and anisotropic magnification from cryo-EM data sets in RELION-3.1. IUCrJ 7, 253-267 (2020).

38. Kucukelbir, A., Sigworth, F. J. \& Tagare, H. D. Quantifying the local resolution of cryo-EM density maps. Nat. Methods 11, 63-65 (2014).

39. Pettersen, E. F. et al. UCSF Chimera-a visualization system for exploratory research and analysis. J. Comput Chem. 25, 1605-1612 (2004).

40. Emsley, P. \& Cowtan, K. Coot: model-building tools for molecular graphics. Acta Crystallogr D. Biol. Crystallogr 60, 2126-2132 (2004).

41. Afonine, P. V. et al. Real-space refinement in PHENIX for cryo-EM and crystallography. Acta Crystallogr D. Struct. Biol. 74, 531-544 (2018).
42. Nicholls, R. A., Long, F. \& Murshudov, G. N. Low-resolution refinement tools in REFMAC5. Acta Crystallogr D. Biol. Crystallogr 68, 404-417 (2012).

43. Brown, A. et al. Tools for macromolecular model building and refinement into electron cryo-microscopy reconstructions. Acta Crystallogr D. Biol. Crystallogr 71, 136-153 (2015).

44. Scheres, S. H. RELION: implementation of a Bayesian approach to cryo-EM structure determination. J. Struct. Biol. 180, 519-530 (2012).

\section{Acknowledgements}

We are grateful to Dr. Dong-Hua Chen for technical support for sample freezing, the cryo-EM facility staff at Columbia University Irving Medical Center (CUIMC) for technical assistance in cryo-EM data acquisition, and Dr. Ya-Ming Hou for the kind gifts of the flexizyme substrates. This work was supported by the US National Institutes of Health (NIH) grants GM011378 and AI047365 to J.D.P.; a Knut and Alice Wallenberg Foundation postdoctoral scholarship to J.W. (KAW 2015.0406); the Intramural Research Program of the NIH (T.E.D.).

\section{Author contributions}

Jinfan W. prepared the cryo-EM samples and performed single-molecule experiments Jing W. and I.S.F. acquired and processed the cryo-EM data and built the structural models; B.-S.S. and J.-R.K. performed the yeast growth and reporter experiments; T.E.D., J.D.P. and I.S.F. supervised the work; Jinfan W., B.-S.S., T.E.D., J.D.P., and I.S.F. wrote the paper with input from all the other authors.

\section{Competing interests}

The authors declare no competing interests.

\section{Additional information}

Supplementary information is available for this paper at https://doi.org/10.1038/s41467 020-18829-3.

Correspondence and requests for materials should be addressed to T.E.D., J.D.P. or I.S.F.

Peer review information Nature Communications thanks the anonymous reviewers for their contribution to the peer review of this work

Reprints and permission information is available at http://www.nature.com/reprints

Publisher's note Springer Nature remains neutral with regard to jurisdictional claims in published maps and institutional affiliations.

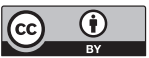

Open Access This article is licensed under a Creative Commons Attribution 4.0 International License, which permits use, sharing, adaptation, distribution and reproduction in any medium or format, as long as you give appropriate credit to the original author(s) and the source, provide a link to the Creative Commons license, and indicate if changes were made. The images or other third party material in this article are included in the article's Creative Commons license, unless indicated otherwise in a credit line to the material. If material is not included in the article's Creative Commons license and your intended use is not permitted by statutory regulation or exceeds the permitted use, you will need to obtain permission directly from the copyright holder. To view a copy of this license, visit http://creativecommons.org/ licenses/by/4.0/

(c) The Author(s) 2020 